\title{
Universiteit
}

Leiden

The Netherlands

\section{Inverse spin switch effects in ferromagnet-superconductor-ferromagnet trilayers with strong ferromagnets}

Rusanov, A.Yu.; Habraken, M.M.P.; Aarts, J.

\section{Citation}

Rusanov, A. Y., Habraken, M. M. P., \& Aarts, J. (2006). Inverse spin switch effects in ferromagnet-superconductor-ferromagnet trilayers with strong ferromagnets. Physical Review $B, 73,060505$. doi:10.1103/PhysRevB.73.060505

Version: $\quad$ Not Applicable (or Unknown)

License: $\quad$ Leiden University Non-exclusive license

Downloaded from: https://hdl.handle.net/1887/44630

Note: To cite this publication please use the final published version (if applicable). 


\title{
Inverse spin switch effects in ferromagnet-superconductor-ferromagnet trilayers with strong ferromagnets
}

\author{
A. Yu. Rusanov, S. Habraken, and J. Aarts \\ Kamerlingh Onnes Laboratory, Leiden University, 2300 RA Leiden, The Netherlands \\ (Received 5 September 2005; revised manuscript received 19 January 2006; published 22 February 2006)
}

\begin{abstract}
In ferromagnet-superconductor-ferromagnet $(F / S / F)$ trilayers where the magnetization directions of the $F$ layers can be controlled separately, it has theoretically been predicted that the antiparallel (AP) configuration can have a higher superconducting transition temperature $T_{c}$ than the parallel $(P)$ configuration. This is the so-called spin switch, which has been found experimentally for the case of weak ferromagnets. Here we show that strong ferromagnets yield the opposite effect. We study the transport properties of $F / S / F$ trilayers with $F=\mathrm{Ni}_{0.80} \mathrm{Fe}_{0.20}$ (Permalloy, Py) and $S=\mathrm{Nb}$, structured in strips of different sizes. Using two different thicknesses for the Py layers, we can switch, in a well-defined way, between the AP and $P$ configurations. In the superconducting transition we find a clear increase of the resistance in the AP state. We ascribe this to enhanced reflection of spin-polarized quasiparticles at the $S / F$ interfaces, which leads to a stronger suppression of superconductivity on the $S$ side.
\end{abstract}

DOI: 10.1103/PhysRevB.73.060505

PACS number(s): 74.78. $-\mathrm{w}, 74.45 .+\mathrm{c}, 85.25 .-\mathrm{j}$

One of the interesting phenomena that is currently searched for in hybrids of superconductors $(S)$ and ferromagnets $(F)$ is the so-called superconducting spin switch effect. Basically, the effect can occur in $F / S / F$ trilayers in which the direction of the magnetization of one $F$ layer can be varied with respect to the other. It was predicted some time ago that, in a current-in-plane (CIP) geometry, and for a thickness of the $S$ layer $d_{S}$ of the order of its superconducting coherence length $\xi_{S}$, the transition temperature $T_{c}^{A P}$ in the antiparallel (AP) state is higher than the one in the parallel $(P)$ state, $T_{c}^{P}{ }^{1,2}$ For particular choices of the different layer thicknesses it should even be possible to find full reentrant behavior, controlled with only a small switching field. ${ }^{3}$ The effect is reminiscent of $F / N / F$ spin valves ( $N$ being a normal metal), with one important difference. In the normal metal spin valves, the resistance is lowest in the parallel configuration, since in terms of a two-spin-current model, it is determined by the spin channel with the smallest resistance. In the superconducting case, the antiparallel configuration yields the lowest (zero) resistance since the Cooper pair samples opposite exchange fields, which are less pair breaking than parallel fields.

Full reentrant behavior has not yet been observed in superconducting spin valves. Two experiments were reported. Both were with a device consisting of weakly ferromagnetic $\mathrm{CuNi}$ and superconducting $\mathrm{Nb}$, and in both cases the reported $T_{c}^{A P}$ was only about $5 \mathrm{mK}$ (Ref. 4) or $2.5 \mathrm{mK}$ (Ref. 5) higher than $T_{c}^{P}$, less than the width of the transition. The smallness of the effect is probably due to the difficulty of producing highly transparent interfaces with these and similar alloys. It is of importance to note that both theory and experiment are performed in the limit of weak exchange field $h_{e x}$, with effectively one diffusion constant for both spin species, very similar amounts of spin-up and spin-down particles, and therefore weak spin-polarization $P_{s}$. The effects of larger $h_{e x}$ or $P_{s}$ are unknown and, at least for the CIP case, difficult to access by theory.

Here we want to show that high spin polarization actually leads to the opposite effect. In trilayer combinations of ferromagnetic permalloy $\left(\mathrm{Ni}_{80} \mathrm{Fe}_{20} ; \mathrm{Py}\right)$ with superconducting $\mathrm{Nb}$, we find that, in the superconducting transition, switching from the $P$ to the AP configuration leads to an increase rather than a decrease of the resistance when measured in a CIP geometry. The effect is strong; it is very similar to recently reported findings on trilayers of ferromagnetic $\mathrm{La}_{0.7} \mathrm{Ca}_{0.3} \mathrm{MnO}_{3}(L)$, where $P_{s}$ is expected to be close to $100 \%$, and superconducting $\mathrm{YBa}_{2} \mathrm{Cu}_{3} \mathrm{O}_{7}(Y),{ }^{6}$ and we offer a similar explanation in terms of reflection of spin-polarized quasiparticles near the interface. ${ }^{7}$ Where the conclusions in Ref. 6 had to be based on indirect evidence for the AP configuration, the use of Py allows us to demonstrate switching effects directly. Together, the experiments based on oxides and on simple metals show that the effect is generic for the limit of high spin polarization.

Samples of $\mathrm{Py} / \mathrm{Nb} / \mathrm{Py}$ were prepared by sputter deposition in an ultrahigh vacuum system. Thick $\mathrm{Nb}$ films have a $T_{c}$ of $9.2 \mathrm{~K}$, similar to the bulk. From the upper critical fields we extract a value for the Ginzburg-Landau (GL) coherence length $\xi_{G L}(0) \approx 13 \mathrm{~nm}$. Using $\xi_{G L}(0)=0.86 \sqrt{\xi_{0} \ell_{N}}$, with the BCS coherence length $\xi_{0} \approx 40 \mathrm{~nm}$, this yields a value for the normal state elastic mean free path $\ell_{N} \approx 5.5 \mathrm{~nm}$. Samples were structured by $e$-beam lithography in bridges of $0.5 \mathrm{~mm} \times 4 \mathrm{~mm}$ ("large" samples) or in bridges of $3 \mu \mathrm{m}$ $\times 20 \mu \mathrm{m}$ ("small" samples). For both large and small samples we used a design in which the contacts were included in the geometry as well as simple bars with gold contacts in order to minimize problems with stray fields from contact pads or arms. Ferromagnetic Py possesses a large spin polarization $[45 \%$ (Ref. 8 ) ], but also shows well-defined magnetization switching at low fields. Care was taken to align the long axis of the bars with the easy axis of magnetization $\hat{e}_{e}$, which is induced by the residual magnetic fields in the sputtering machine. Magnetic fields were applied in the plane of the sample, along the bars and therefore along $\hat{e}_{e}$. We also made use of the fact that the coercive field $H_{c}$ of the Py layers depends on their morphology as well as on 


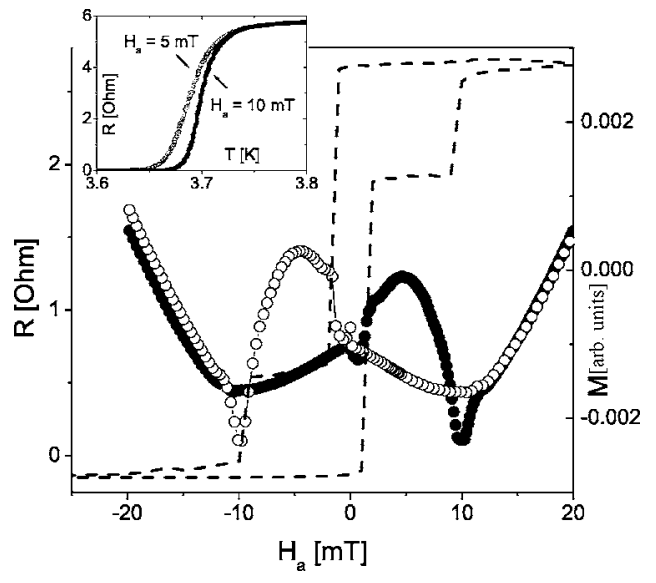

FIG. 1. Resistance $R$ vs applied field $H_{a}$ of a large sample $s / \mathrm{Py}(50) / \mathrm{Nb}(25) / \mathrm{Py}(20)$ at $3.66 \mathrm{~K}$. Filled (open) symbols, forward (backward) sweep. Dashed, magnetization $M$ vs $H_{a}$ at $5 \mathrm{~K}$ of a similar but unstructured sample. Inset: $R$ vs temperature $T$ at $5 \mathrm{mT}$ in the $P$ state and at $10 \mathrm{mT}$ in the domain state.

thickness. We consistently find that a thicker Py layer deposited on the substrate has a lower value of $H_{c}$ than a thinner layer deposited on top of the $\mathrm{Nb}$ layer. In this way it is quite possible to have well-defined $P$ and AP regimes. Typically, we used $50 \mathrm{~nm}$ for the Py bottom layer and $20 \mathrm{~nm}$ for the Py top layer, which yields coercive or switching fields around $1-2 \mathrm{mT}(50 \mathrm{~nm})$ and $8-10 \mathrm{mT}(20 \mathrm{~nm})$. For the Nb layer, the smallest thickness $d_{N b}$ was around $25 \mathrm{~nm}$, which yields transition temperatures around $4 \mathrm{~K}$ and is already close to the critical thickness $d_{c r}$ for the trilayer.

Figure 1 shows a compilation of different measurements on a sample $s / \mathrm{Py}(50) / \mathrm{Nb}(25) / \mathrm{Py}(20)$ (with $s$ the substrate and the numbers the layer thickness in nanometer). The magnetization was measured at $5 \mathrm{~K}$ on the unstructured sample by superconducting quantum interference device (SQUID) magnetometry. The switching of the layers is well defined and from the jump sizes it can be seen that the 50-nm layer switches at $\pm H_{c, 50} \approx 1.5 \mathrm{mT}$, and the 20 -nm layer at $\pm H_{c, 20}$ $\approx 9.5 \mathrm{mT}$, leaving a large field range for the AP state. For transport measurements, the sample was structured as a large bar with gold contacts and showed a resistive transition around $3.7 \mathrm{~K}$ with a width $\Delta_{t r}$ of $100 \mathrm{mK}$. The resistance $R$ was measured as function of the in-plane field $H_{a}$ at a temperature of $3.66 \mathrm{~K}$, as shown in Fig. 1. Starting at high fields, $R$ decreases until $+H_{c, 20}$ where it starts to rise slowly. At $-H_{c, 50}$ a small but clear upward jump occurs. This is the field where the alignment of the Py layers becomes AP. In this regime $R$ rises further to a peak, followed by a steep decrease to a dip at around $-H_{c, 20}$. Now the sample is in the $P$ state and $R$ starts to rise slowly again. The behavior is mirrored in increasing fields. The strong peaks in the resistance therefore appear to be connected to the AP alignment, just as in the case of the $L / Y / L$ trilayers of Ref. 6 . The dips at $\pm H_{c, 20}$ are well known, produced by the magnetic domains which occur around the Py coercive field in the 20-nm layer. They can also be found in Py/Nb bilayers, and are due to a lower averaged exchange field sensed by the Cooper pair, as we demonstrated previously. ${ }^{9}$ For the $50-\mathrm{nm}$ layer they also

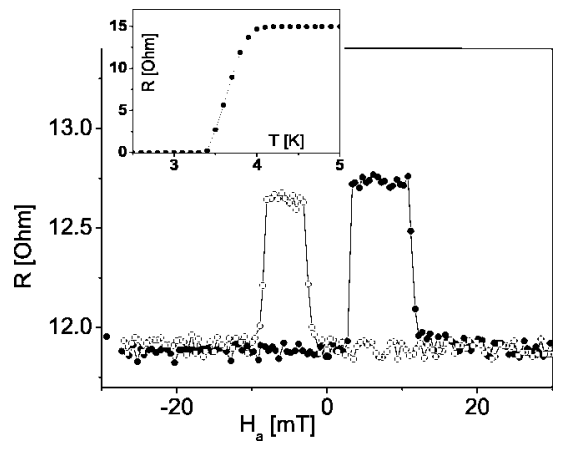

FIG. 2. Resistance $R$ vs applied field $H_{a}$ of a bridge of $3 \mu \mathrm{m}$ $\times 20 \mu \mathrm{m}$ (contacts included) of a sample $s / \mathrm{Py}(50) / \mathrm{Nb}(26) / \mathrm{Py}(20)$ at $3.8 \mathrm{~K}$. Filled (open) symbols, forward (backward) sweep. Inset: $R$ vs temperature $T$.

should be present, and a small dip is actually observed in backward sweep at $+1.5 \mathrm{mT}$, but it is masked by the increase of resistance resulting from the $P \rightarrow \mathrm{AP}$ switch. The effect of the domain state on $T_{c}$ is shown in the insert, where $R(T)$ is given at two different fields; at $5 \mathrm{mT}$ coming from high field (sample in the $P$ state), and at $10 \mathrm{mT}$ coming from low fields (sample in the domain state). The difference in the temperature where zero resistance is reached is at most $30 \mathrm{mK}$, similar to the earlier findings. Finally, the rise in $R$ at $+H_{c, 20}$ when coming from high fields may appear puzzling, since no switching takes place at this field. Domain formation, however, already does set in: close inspection of $M(H)$ shows that decrease already starts before $H_{c, 50}$ is reached, and this should lead to small amounts of AP orientations.

Upward jumps were never found in the bilayers, ${ }^{9}$ and the data therefore suggest that it is a trilayer effect in which the AP state shows larger resistance than the $P$ state, but that for the large sample the behavior is sluggish because of domain effects. Next we consider some much smaller samples. Figure 2 shows data on a sample $s / \mathrm{Py}(50) / \mathrm{Nb}(26) / \mathrm{Py}(20)$ with a bridge of $3 \mu \mathrm{m} \times 20 \mu \mathrm{m}$ and contacts included. The transition (see inset) is quite broad, $\Delta_{t r} \approx 600 \mathrm{mK}$ and the measurement is taken at $3.80 \mathrm{~K}$, close to the onset at $4 \mathrm{~K}$. After correcting for a small offset field, the values for $\pm H_{c, 50}$ and $\pm H_{c, 20}$ are 2.7 and $10 \mathrm{mT}$, respectively. The switching behavior is now perfectly well defined. Also noticeable is the absence of the dips that we ascribed to the domains. This is again in agreement with our earlier observations on small bilayers and due to the fact that no stable domain state is formed in these small samples during the switching. ${ }^{9}$ Also note that the bilayers do not show such switching. Trilayer samples with similar thicknesses show the same behavior, although the switching is not always a perfect one-step process; sometimes, several steps (both up and down) can be seen. This may not be surprising, since the properties of Py, and the direction of the easy axis are very sensitive to the preparation conditions. Next we increase $d_{N b}$. The transition width now gradually decreases. Figure 3 shows data on a sample $s / \mathrm{Py}(50) / \mathrm{Nb}(60) / \mathrm{Py}(20)$ with $\Delta_{t r} \approx 100 \mathrm{mK}$ and a normal state resistance of $9.89 \Omega$. In this case, the sample was a simple bar of $3 \mu \mathrm{m} \times 20 \mu \mathrm{m}$ with Au contacts, which we show to make clear that the effect can be found with different contact geometries. The behavior of $R\left(H_{a}\right)$ is shown 


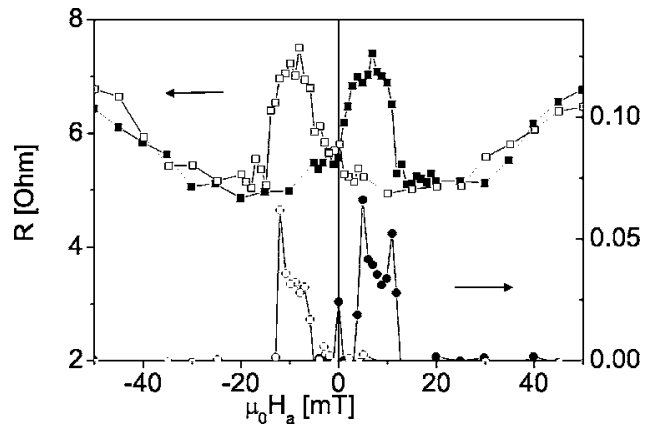

FIG. 3. Resistance $R$ vs applied field $H_{a}$ of a $2 \mu \mathrm{m} \times 20 \mu \mathrm{m}$ bridge of a sample $s / \mathrm{Py}(50) / \mathrm{Nb}(60) / \mathrm{Py}(20)$ at $7.46 \mathrm{~K}$ (squares) and at $7.40 \mathrm{~K}$ (circles). Filled (open) symbols, forward (backward) sweep.

at $T=7.46 \mathrm{~K}$, halfway the transition, and at $T=7.40 \mathrm{~K}$, close to the bottom. The switching behavior is still sharp and clear, quite similar to the previous sample. Note that the size of the resistance variation has become much smaller at the lower temperature. For samples with $d_{N b}=80,90 \mathrm{~nm}$ and $\Delta_{t r}$ $\leqslant 50 \mathrm{mK}$, the effect became very small. Above $T_{c}, R\left(H_{a}\right)$ of the samples only shows tiny dips around $\pm H_{c, 50}$, which are due to the anisotropic magnetoresistance (AMR) effect for configurations where the current is parallel to the applied magnetic field. ${ }^{10}$

The observed effects are therefore due to the onset of the superconducting state. For all geometries, in the resistive transition, the AP state has a higher resistance than the $P$ state, which is opposite to the effects predicted ${ }^{1,3}$ and observed for weak ferromagnets, ${ }^{4,5}$ but similar to the observations of Ref. 6. In terms of the magnetoresistance $(M R)$ ratio $\Delta R / R=\left(R_{A P}-R_{P}\right) / R_{P}$, the effect becomes quite large due to the decreasing value of $R$, but for the physics it is more relevant to note that in terms of the normal-state resistance of the samples, it is a fraction of the order of 5\%-10\%, at least in the upper half of the transition. It is then of interest to compare the results of these CIP measurements to the results of current-perpendicular-to-plane (CPP) measurements on stacks of $\mathrm{Py} / \mathrm{Nb} / \mathrm{Py}$, performed by $\mathrm{Gu}$ et al. ${ }^{11}$ for different thicknesses $d_{N b}$ of the $\mathrm{Nb}$ layer in a range between 30 and $100 \mathrm{~nm}$. In the CPP case, a small $(1 \%)$ positive $\left(R_{A P}>R_{P}\right)$ effect was present in the normal state, which persisted below $T_{c}$. However, it was found to decrease with decreasing temperature, which is different from our CIP data that show an initial increase of the $M R$ ratio (from 0 in the normal state). A basic explanation of the CPP data was given in terms of the diffusion of spin-polarized quasiparticles (qp) with energies $E_{q p}$ below the gap. The $M R$ effect in the normal state is due to the standard mechanism of increased spin scattering in the AP configuration. This becomes smaller through spin memory loss controlled by the spin diffusion length $\ell_{s d}$, but when the intermediate layer is a superconductor, where spin is carried only by quasiparticles and not by Cooper pairs, $M R$ ratio will also decrease due to the loss of quasiparticles to the condensate, which is controlled by the qp diffusion length $\xi_{q p}{ }^{12}$ From the temperature dependence of $\Delta R$, a value of $\xi_{q p} \approx 16.5 \mathrm{~nm}$ was found, very close to $\xi_{G L}(0)$ $\approx 13 \mathrm{~nm}$ of the $\mathrm{Nb}$. This is actually not surprising. It was already shown by Blonder, Tinkham, and Klapwijk that the characteristic decay of evanescent quasiparticles with energies inside the gap (the current-to-supercurrent conversion length) is given by $1.22 \xi_{G L}(T) .{ }^{13}$ The number also shows that spin loss by spin scattering only plays a minor role, since $\xi_{G L}(0) \ll \ell_{s d} \approx 50 \mathrm{~nm} .{ }^{11}$ This analysis was confirmed in recent theoretical work by Yamashita et al. ${ }^{14}$ who considered Andreev reflections and direct transmission of spin-polarized quasiparticles in $F / S / F$ systems. They found slightly larger values for $\xi_{q p}$, and also that $\xi_{q p}$ decreases with increasing $d_{N b}$. This was ascribed to the proximity effect in this allmetal system, which suppresses the average gap as long as $d_{N b}$ is comparable to $\xi_{G L}$.

Translating this description to our CIP case, the first thing to note is the different $M R$ in the normal state. This is easily explained. In CPP, for diffusive systems, the dependence of $\Delta R / R$ on the spacer thickness $d_{N}$ is $\propto e^{-d_{N} / 2 \ell_{s d}} .{ }^{15}$ If $M R$ effects are present in the $\mathrm{Py} / \mathrm{Nb} / \mathrm{Py}$ system, they can be witnessed for the range of thicknesses used in the CPP experiment. On the other hand, in CIP the attenuation is $\propto e^{-d_{N} / \ell_{N}}$, with $\ell_{N}$ the elastic mean free path of the normal metal. ${ }^{16,17}$ For our $\mathrm{Nb}$, we estimated before that $\ell_{N} \approx 5.5 \mathrm{~nm}$, much smaller than the spacer thickness of $25 \mathrm{~nm}$. So, if $M R$ effects are found in CPP, they will not be observed in CIP in the same thickness range, and the two sets of results are not inconsistent. In the superconducting transition the situation is different, since then quasiparticles appear with a much longer range because of the divergence close to $T_{c}$. We now offer a similar line of reasoning as Ref. 6. Current is flowing in the plane of the films, there is no voltage difference perpendicular to the layers, so electrons scattering out of the $F$ layer turn into spin-polarized quasiparticles with energies around or below the gap. A fraction of these will scatter back into the $F$ layer; another (smaller) fraction will diffuse across the $S$ layer. Of this fraction, a larger part experiences reflection at the other interface in the AP configuration than in the $P$ configuration, leading to a larger number of quasiparticles on the $S$ side for the AP case than for the $P$ case. Although the spin determines this reflection process, the result should probably not be called spin accumulation since there is no net charge or spin transport through the interface, ${ }^{15}$ in contrast to the CPP case. The larger number of quasiparticles on the $S$ side for the AP case translates self-consistently into a gap suppression on the $S$ side. Note that this is the case even if these quasiparticles diffuse back all the way into the original $F$ layer. The gap suppression then is observed as an increased resistance.

Based on the magnitude of the switching, we can estimate the size of the layer where this takes place for temperatures close to $T_{c}$, at the top of the transition, where the order parameter profile is still flat. In the normal state, the specific resistances are $\rho_{N b}=7.5 \mu \Omega \mathrm{cm}$ for the $\mathrm{Nb}$ and $\rho_{\text {Py }}$ $=30 \mu \Omega \mathrm{cm}$ for the Py. The contribution to the resistance of the $\mathrm{Nb}$ layer in a sample $s / \mathrm{Py}(50) / \mathrm{Nb}(25) / \mathrm{Py}(20)$ is therefore $40 \%$. Assuming that the full variation in $\Delta R / R_{N} \approx 10 \%$ is due to extra resistance in the $\mathrm{Nb}$ layer, this corresponds to $25 \%$ of the $\mathrm{Nb}$ layer, which is $6 \mathrm{~nm}$, or $3 \mathrm{~nm}$ on each side. This rough estimate suggests a suppressed layer with a thickness of the order of $\ell_{N}$. Finding the exact dependence of $\Delta R$ on temperature and thickness needs more elaborate model- 
ing. The number of quasiparticles decreases with decreasing temperature and increasing gap size, and this partly explains the smallness of $\Delta R$ for the sample with $d_{N b}=50 \mathrm{~nm}$ near the bottom of the transition, but it is also a function of thickness. Close to $d_{c r}$, which is around $2 \xi_{G L}(0)$, the order parameter stays flat, but with increasing thickness it will grow more strongly in the middle of the layer and act as a bottleneck for the evanescent quasiparticles, since their accessible energy range becomes smaller. This should lead to a decreasing transition width, as witnessed, but also to different behavior of $\Delta R$ as function of reduced temperature $T / T_{c}$. Also the effect of the spin diffusion length will start to play a role around $d_{N b} \approx 50 \mathrm{~nm} \approx \ell_{s d}$, but because of the above argument it cannot be claimed that the loss of $M R$ effects for our thickest samples is simply due to the loss of spin.

In conclusion, our observations put a different perspective on the feasibility of the superconducting spin switch. Close to $T_{c}$, spin switch effects can be found with weak ferromagnets but the difficulty of obtaining highly transparent interfaces when using strongly disordered alloys may preclude full switching. Increasing the polarization, however, leads to a competing effect, namely the increased quenching of the superconductivity when the AP configuration reflects more spin-polarized quasiparticles back into the superconductor than the $P$ configuration. Note that both mechanisms are not mutually exclusive since one depends on the quasiparticles and the other on the Cooper pairs. We also have made clear the differences between CIP and CPP experiments. Finally, our observations should be of importance for the reproducibility and interpretation of data from $S / F$ multilayers with strong magnets in general. In many reported experiments on $T_{c}$ variations, the magnetization state of the sample is undefined, and in particular reentrant effects close to the critical thickness might be affected by the domain state of the sample.

We thank J. Santamaria, C. Bell and M. Flokstra for discussions, and M. Hesselberth for help in sample preparation. The work is part of the research program of the Stichting "F.O.M.," which is financially supported by NWO. The ESFprograms "Pishift" and "Thiox" are acknowledged for providing an invaluable forum for discussing the preliminary results.
${ }^{1}$ A. I. Buzdin, A. V. Vedyayev, and N. V. Ryzhanova, Europhys. Lett. 48, 686 (1999).

${ }^{2}$ I. Baladié, A. Buzdin, N. Ryzhanova, and A. Vedyayev, Phys. Rev. B 63, 054518 (2001).

${ }^{3}$ L. R. Tagirov, Phys. Rev. Lett. 83, 2058 (1999).

${ }^{4}$ J. Y. Gu, C.-Y. You, J. S. Jiang, J. Pearson, Ya. B. Bazaliy, and S. D. Bader, Phys. Rev. Lett. 89, 267001 (2002).

${ }^{5}$ A. Potenza and C. H. Marrows, Phys. Rev. B 71, 180503(R) (2005).

${ }^{6}$ V. Pena, Z. Sefrioui, D. Arias, C. Leon, J. Santamaria, J. L. Martinez, S. G. E. teVelthuis, and A. Hoffmann, Phys. Rev. Lett. 94, 057002 (2005).

${ }^{7}$ S. Takahashi, H. Imamura, and S. Maekawa, Phys. Rev. Lett. 82, 3911 (1999).

${ }^{8}$ J. S. Moodera, J. Nowak, and R. J. M. van de Veerdonk, Phys. Rev. Lett. 80, 2941 (1998).

${ }^{9}$ A. Yu. Rusanov, M. Hesselberth, J. Aarts, and A. I. Buzdin, Phys. Rev. Lett. 93, 057002 (2004).
${ }^{10}$ R. O'Handley, Modern Magnetic Materials (Wiley, New York, 2000), p. 574.

${ }^{11}$ J. Y. Gu, J. A. Caballero, R. D. Slater, R. Loloee, and W. P. Pratt, Phys. Rev. B 66, 140507(R), 2002.

${ }^{12}$ In Ref. 11 the notation $\ell_{q p}$ was used for what was called the quasiparticle penetration depth. We prefer $\xi_{q p}$ in order to stress the superconducting nature of the length scale, which is reflected in its divergence at $T_{c}$.

${ }^{13}$ G. E. Blonder, M. Tinkham, and T. M. Klapwijk, Phys. Rev. B 25, 4515 (1982).

${ }^{14}$ T. Yamashita, H. Imamura, S. Takahashi, and S. Maekawa, Phys. Rev. B 67, 094515 (2003).

${ }^{15}$ T. Valet and A. Fert, Phys. Rev. B 48, 7099 (1993).

${ }^{16}$ V. S. Speriosu, J. P. Nozières, B. A. Gurney, B. Dieny, T. C. Huang, and H. Lefakis, Phys. Rev. B 47, 11579 (1993).

${ }^{17} \mathrm{R}$. Coehoorn in Magnetic Multilayers and Giant Magnetoresistance, edited by U. Hartmann, Springer Series in Surface Sciences Vol. 37 (Springer, Berlin, 2000). 